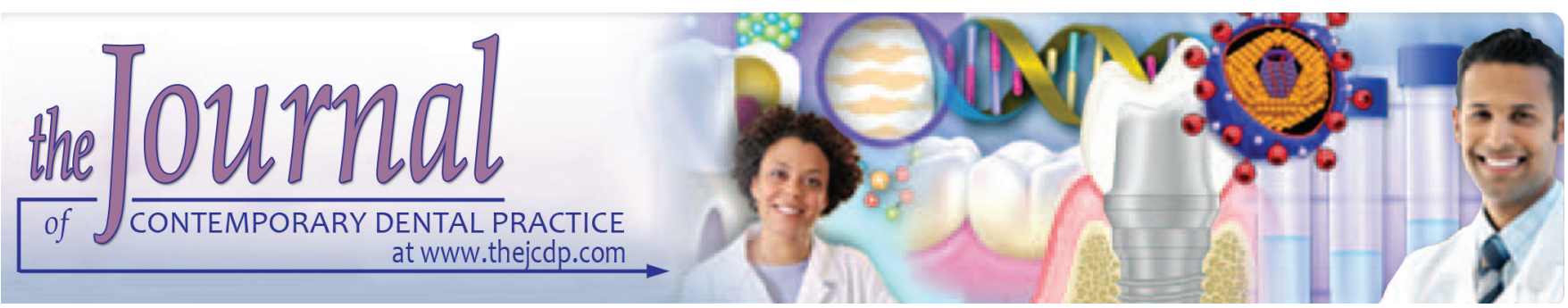

\title{
Assessment of Lipid Peroxidation Levels and Total Antioxidant Status in Chronic and Aggressive Periodontitis Patients: An in vivo Study
}

${ }^{1}$ Vivek Tripathi, ${ }^{2}$ Sahib T Singh, ${ }^{3}$ Vivek Sharma, ${ }^{4}$ Ashish Verma, ${ }^{5}$ Chetan D Singh, ${ }^{6}$ Jaspreet S Gill

\begin{abstract}
Introduction: Periodontitis is a common problem affecting a significant population of the world. For the assessment of oxidative stress of an individual, total oxidation status (TOS) and total antioxidant capacity (TAOC) are the significant biomarkers. Hence, we planned the present study to assess malondialdehyde (MDA), TOS, TAOC levels, and oxidative stress index (OSI) in generalized aggressive periodontitis (GP) and chronic periodontitis $(\mathrm{CP})$ patients.
\end{abstract}

Materials and methods: The present study included assessment of $40 \mathrm{CP}$ patients, $40 \mathrm{GP}$ patients, and 40 healthy controls. Clinical assessment of all the subjects was done by measuring the probing depth (PD), clinical attachment (CL), gingival index (GI), gingival bleeding index (GBI), and plaque index (PI). Salivary and serum samples were taken and assessed by standard procedures as described previously in the literature. All the values were assessed and compared.

Results: Significant results were obtained while comparing all the periodontal parameters in between various study groups. Mean serum MDA levels in the CP, GP, and control group were found to be $0.68,0.65$, and $0.61 \mu \mathrm{M}$ respectively. Statistically nonsignificant results were obtained while comparing the serum MDA levels in between the three study groups. Significant results were obtained while comparing the mean serum and salivary TOS values, TAOC values, and OSI in between various study groups.

Conclusion: In periodontitis patients, oxidative stress was significantly higher in comparison with healthy subjects.

\footnotetext{
${ }^{1}$ Department of Periodontics, Azamgarh Dental College \& Hospital, Azamgarh, Uttar Pradesh, India

2,4,5 Department of Periodontology, Sri Guru Ram Das Institute of Dental Sciences \& Research, Amritsar, Punjab, India

${ }^{3,6}$ Department of Periodontology, Desh Bhagat Dental College \& Hospital, Fatehgarh Sahib, Punjab, India

Corresponding Author: Vivek Tripathi, Department of Periodontics, Azamgarh Dental College \& Hospital, Azamgarh Uttar Pradesh, India, Phone: +919501544877, e-mail: drvivektripathi12@gmail.com
}

Clinical significance: Oxidative parameters do play a significant role in the pathologic profile of periodontitis.

Keywords: Chronic periodontitis, Generalized aggressive periodontitis, Oxidative stress.

How to cite this article: Tripathi V, Singh ST, Sharma V, Verma A, Singh CD, Gill JS. Assessment of Lipid Peroxidation Levels and Total Antioxidant Status in Chronic and Aggressive Periodontitis Patients: An in vivo Study. J Contemp Dent Pract 2018;19(3):287-291.

\section{Source of support: Nil}

Conflict of interest: None

\section{INTRODUCTION}

In recent past years, saliva has become a routine component of diagnostic techniques in the field of clinical research and diagnostic pathology. Its noninvasive nature, easy method of collection, and ease of availability make it more advantageous over other diagnostic fluids. ${ }^{1,2}$ Before establishing it in routine clinical practice, its specificity and sensitivity have to be established. The two most common diseases of the oral cavity are periodontitis and dental caries. ${ }^{3}$ Biomarkers are a group of parameters that allow early detection and help in establishment of diagnosis and prognosis of a disease. For the diagnosis of oxidative stress, optimal biomarkers should be both sensitive and specific. ${ }^{4,5}$

Lipid peroxidation (LPO) is most frequently studied in terms of MDA levels. Total oxidation status and TAOC are the significant biomarkers for the assessment of oxidative stress of an individual. Another important oxidative parameter for assessing the oxidative stress includes OSI. In the literature, the difference and similarity in the oxidative profile of GP and CP are not clearly established. ${ }^{6-8}$ Hence, we planned the present study to assess the oxidative stress biomarkers in patients with CP and GP. 


\section{MATERIALS AND METHODS}

The present study was conducted in the Department of Periodontology of the Dental Institute and included assessment of 120 subjects. Ethical approval was taken from Institutional Ethical Committee and written consent was obtained after explaining in detail the entire research protocol. Of 120 subjects included in the present study, 40 subjects were of $\mathrm{CP}, 40$ subjects were of GP, and the remaining 40 subjects were taken as healthy controls. All the groups consisted of equal number of males and females. All the patients included in the present study reported to the college outpatient department for checkup of routine periodontal problems. Following the criteria given by the American Academy of Periodontology, complete clinical and radiographic evaluation of all the subjects was done for the diagnosis of $\mathrm{CP}$ and GP. ${ }^{9,10}$ The following clinical and periodontal criteria were used for establishing diagnosis of both the study groups.

\section{Chronic Periodontitis Group}

Patients having more than or equal to $30 \%$ of periodontal bone loss, with teeth having level of CL of $\geq 5 \mathrm{~mm}$, along with periodontal $\mathrm{PD}$ of $\geq 5 \mathrm{~mm}$ at single or multiple sites on teeth at more than one site of all tooth quadrants.

\section{Generalized Aggressive Periodontitis Group}

Patients within the age group of 18 to 40 years, having $\geq 20$, having CL of $\geq 6 \mathrm{~mm}$ and $\mathrm{PD}$ of $\geq 6 \mathrm{~mm}$ at one or multiple sites of $\geq 12$ teeth.

\section{Control Group}

Control group consisted of subjects with healthy periodontal status as characterized by no history of periodontal diseases, with a PD of $\leq 3 \mathrm{~mm}, \mathrm{CL}$ of $\leq 1$, good oral hygiene, and absence of gingival inflammation.

Exclusion criteria for the present study were as follows:

- Patients $<18$ years of age,

- Patients with a history of any systemic illness,

- Patients with any known drug allergy,

- Patients who had taken any form of antibiotic therapy, or anti-inflammatory therapy in the past 3 months,

- Patients with smoking habit.

Clinical assessment of all the subjects was done by measuring the PD, CL, GI, GBI, and PI based on criteria previously described in the literature. All the examination procedures were performed by a single examiner. ${ }^{11-13}$

\section{Collection of Salivary and Serum Samples}

After performing the clinical measurements, 48 hours later, the salivary samples were collected in the morning after overnight fasting. In the morning, on the day of collection of samples, the patients were instructed not to eat or drink anything until the sample was taken. In the present study, unstimulated salivary samples were used. Collection of salivary sample was done over a 5 minute procedure, and the patients were instructed to let the pooling of saliva in the floor of the mouth followed by draining to a collection tube, whenever necessary. Patients were prohibited for swallowing of the saliva for calculation of salivary flow rates (SFRs). By dividing the quantity of saliva obtained, with time, we obtained SFR. ${ }^{14}$ Centrifugation of salivary sample was done at $4,000 \mathrm{~g}$ for 10 minutes at $4^{\circ} \mathrm{C}$. This was followed by storage of the samples in the storage vials. ${ }^{15}$ For collection of venous blood, plain tubes were used, which were maintained at $4^{\circ} \mathrm{C}$ for half an hour followed by centrifugation. Cryogenic vials were used for storing the serum samples. Estimation of salivary and serum MDA levels was done by MDA (LPO) assay, previously described by Young and Trimble. ${ }^{16}$ Method described by Ere ${ }^{17}$ was used for the evaluation of serum and salivary TOS levels. For evaluation of TAOC salivary and serum levels, enzymelinked immunosorbent assay (ELISA) was used as per manufacturer's instructions. For the calculation of OSI, we used the percentage ratio of TOS to TAOC. ${ }^{17}$

All the results were compiled and analyzed in Statistical Package for the Social Sciences software. Chisquared test, Student's t-test, and Mann-Whitney U-test were used for the assessment of level of significance; $\mathrm{p}<0.05$ was taken as significant.

\section{RESULTS}

In the present study, we evaluated a total of 120 subjects and divided them broadly into three study groups: $\mathrm{CP}$ group, GP group, and the control group. The mean $\mathrm{PD}$ in $\mathrm{CP}, \mathrm{GP}$, and control group was found to be 3.75, 4.62 , and $1.02 \mathrm{~mm}$ respectively. Significant results were obtained while comparing all the periodontal parameters in between various study groups $(\mathrm{p}<0.05)$ (Table 1$)$. However, we did not observe any significant difference in the SFR of the subjects of various study groups ( $p>0.05)$.

Mean serum MDA levels in the CP, GP, and control group were found to be $0.68,0.65$, and $0.61 \mu \mathrm{M}$ respectively (Table 2). Statistically nonsignificant results were obtained while comparing the serum MDA levels in between the three study groups ( $p>0.05$ ). Comparative evaluation of serum and salivary TOS levels in between various study groups is highlighted in Table 3. Mean value of serum TOS in the $\mathrm{CP}, \mathrm{GP}$, and the control group was found to be $17.5,22.1$, and $14.12 \mu \mathrm{M} \mathrm{H}_{2} \mathrm{O}_{2}$ equivalent respectively. Significant results were obtained while comparing the mean serum and salivary TOS values in 
Table 1: Comparative evaluation of various clinical periodontal parameters among subjects of all the study groups

\begin{tabular}{|c|c|c|c|c|}
\hline Parameter & Groups & Median & $\begin{array}{l}\text { Chi-square } \\
\text { value }\end{array}$ & $p$-value \\
\hline \multirow[t]{3}{*}{$\mathrm{PD}(\mathrm{mm})$} & $\mathrm{CP}$ & $3.75^{\#^{\wedge}}$ & 84.63 & $0.02^{*}$ \\
\hline & GP & $4.62^{\#}$ & & \\
\hline & Control & 1.02 & & \\
\hline \multirow[t]{3}{*}{$\mathrm{CL}(\mathrm{mm})$} & $\mathrm{CP}$ & $4.19^{\#^{\wedge}}$ & 86.14 & $0.03^{*}$ \\
\hline & GP & $5.20^{\#}$ & & \\
\hline & Control & 0.83 & & \\
\hline \multirow[t]{3}{*}{ GI } & $\mathrm{CP}$ & $1.53^{\#^{\wedge}}$ & 84.22 & $0.01^{*}$ \\
\hline & GP & $2.31^{\#}$ & & \\
\hline & Control & 0 & & \\
\hline \multirow[t]{3}{*}{ GBI } & $\mathrm{CP}$ & $1.52^{\#^{\wedge}}$ & 83.12 & $0.02^{*}$ \\
\hline & GP & $2.42^{\#}$ & & \\
\hline & Control & 0 & & \\
\hline \multirow[t]{3}{*}{$\mathrm{Pl}$} & $\mathrm{CP}$ & $1.02^{\#^{\wedge}}$ & 79.25 & $0.01^{*}$ \\
\hline & GP & $2.08^{\#}$ & & \\
\hline & Control & 0 & & \\
\hline \multirow[t]{3}{*}{ SFR } & $\mathrm{CP}$ & 0.38 & 5.25 & 0.25 \\
\hline & GP & 0.40 & & \\
\hline & Control & 0.37 & & \\
\hline
\end{tabular}

*Significant; "\# significant difference in comparison with the control group; ^significant difference in comparison with the GP group

between various study groups $(\mathrm{p}<0.05)$. Comparative evaluation of serum and salivary TAOC levels in between various study groups is shown in Table 4 . Mean serum TAOC values in the $\mathrm{CP}, \mathrm{GP}$, and control group subjects were found to be 1.10,1.04, and $1.50 \mathrm{mM}$ Trolox equivalent respectively. In the $\mathrm{CP}, \mathrm{GP}$, and the control group, the mean salivary TAOC values were found to be $0.60,0.52$, and $0.78 \mathrm{mM}$ Trolox equivalent respectively. We observed

Table 2: Comparative evaluation of serum and salivary MDA levels between various study groups

\begin{tabular}{lllll}
\hline & & \multicolumn{3}{c}{ Chi-square } \\
Parameter & Groups & Mean & value & $p$-value \\
\hline Serum MDA $(\mu \mathrm{M})$ & CP & 0.68 & 0.702 & 0.336 \\
& GP & 0.65 & & \\
& Control & 0.61 & & \\
Salivary MDA $(\mu \mathrm{M})$ & CP & 0.16 & 35.02 & $0.04^{*}$ \\
& GP & 0.16 & & \\
& Control & 0.07 & & \\
\hline
\end{tabular}

*Significant

Table 4: Comparative evaluation of serum and salivary TAOC levels between various study groups

\begin{tabular}{lllll}
\hline & & Mean & Chi-square \\
Parameter & Groups & value & value & $p$-value \\
\hline Serum TAOC (mM & GP & 1.10 & 71.25 & $0.03^{*}$ \\
Trolox equivalent) & GP & 1.04 & & \\
& Control & 1.50 & & \\
Salivary TAOC (mM & CP & 0.60 & 26.35 & $0.04^{*}$ \\
Trolox equivalent) & GP & 0.52 & & \\
& Control & 0.78 & & \\
\hline
\end{tabular}

*Significant significant difference while comparing the mean serum and salivary TAOC values in between various study groups $(p<0.05)$. Significant results were obtained while comparing the serum and salivary OSI in between various study groups (Table 5).

\section{DISCUSSION}

One of the common health problems affecting approximately $10 \%$ of the global population is periodontitis. There exists considerable variation in its prevalence due to variation in both demographic and personal details of the area population. Reactive oxygen species have arisen as significant signaling molecules in different cellular processes. These molecules are instigated from molecular oxygen and principally yield cellular damage, if not neutralized by antioxidant substances. Their formation is a crucial constituent of the host response to a number of noxious stimuli, including bacteria. Oxidative damage can be best predicted by LPO biomarkers. ${ }^{78}$ Hence, we planned the present study to assess the oxidative stress biomarkers in patients with $\mathrm{CP}$ and GP.

In the present study, we observed significant differences while comparing the periodontal parameters in between the three study groups $(\mathrm{p}<0.05)$ (Table 1$)$. Attachment loss was significantly more in patients of GP study group. Our results were in correlation with the results obtained by Baltacığlu et $\mathrm{al}^{18}{ }^{18}$ who also reported similar findings in their study. In a prospective study, Akalin et $\mathrm{al}^{14}$ investigated the MDA levels and TOS values in saliva, serum, and gingival crevicular

Table 3: Comparative evaluation of serum and salivary TOS levels between various study groups

\begin{tabular}{lllll}
\hline & \multicolumn{3}{c}{ Mean } & Chi-square \\
Parameter & Groups & value & value & $p$-value \\
\hline Serum TOS $\left(\mu \mathrm{M} \mathrm{H}_{2} \mathrm{O}_{2}\right.$ & $\mathrm{CP}$ & 17.5 & 60.25 & $0.01^{*}$ \\
equivalent $)$ & $\mathrm{GP}$ & 22.1 & & \\
& Control & 14.12 & & \\
Salivary TOS $(\mu \mathrm{M}$ & $\mathrm{CP}$ & 6.56 & 81.25 & $0.02^{*}$ \\
$\mathrm{H}_{2} \mathrm{O}_{2}$ equivalent) & GP & 7.75 & & \\
& Control & 4.30 & & \\
& & & & \\
\hline
\end{tabular}

*Significant

Table 5: Comparative evaluation of serum and salivary OSI levels between various study groups

\begin{tabular}{|c|c|c|c|c|}
\hline Parameter & Groups & $\begin{array}{l}\text { Mean } \\
\text { value }\end{array}$ & $\begin{array}{l}\text { Chi-square } \\
\text { value }\end{array}$ & $p$-value \\
\hline \multirow[t]{3}{*}{ Serum OSI } & $\mathrm{CP}$ & 1.6 & 44.25 & $0.02^{*}$ \\
\hline & GP & 2.20 & & \\
\hline & Control & 0.92 & & \\
\hline \multirow[t]{3}{*}{ Salivary OSI } & $\mathrm{CP}$ & 1.7 & 39.52 & $0.01^{*}$ \\
\hline & GP & 0.62 & & \\
\hline & Control & 1.30 & & \\
\hline
\end{tabular}

*Significant 
fluid (GCF) of the CP patients. They evaluated a total of $36 \mathrm{CP}$ patients and 28 control subjects and did their clinical assessment. Sampling was done in all these subjects and evaluation of MDA and TOS levels was done by liquid chromatography. While assessing the mean serum MDA levels in between the study group and the control group, they did not observe any significant difference. However, significant difference was observed while comparing the salivary and GCF MDA level in between the study group and control group. However, compared with the control group, the authors observed significantly higher values of serum and salivary TOS in the $\mathrm{CP}$ group. From the above results, the authors concluded that a key role is played by LPO and TOS in the pathology of periodontitis. Furthermore, significant results were obtained while comparing the mean serum TOS, TAOC, and OSI levels in between the study group and the control group (Tables 4 and 5). A significant product of LPO is MDA. In a study conducted by Baltacioğlu et $\mathrm{al}_{1}{ }^{18}$ levels of MDA, TOS, TAOC, and OSI were evaluated in the serum and saliva of periodontitis patients. They assessed a total of 98 patients and divided them into three study groups; $\mathrm{CP}$ group with 33 patients, GP group with 35 patients, and healthy control group with 30 patients. Liquid chromatography method was used for the estimation of MDA, TOS, and TAOC levels, while they used calorimetric method for clinical assessment of the samples. For the calculation of the OSI values, they used the formulae "TOS/TOAC $\times 100$ " for the estimation of mean OSI values. Significantly higher levels of salivary MDA and serum and salivary TOS and OSI were observed in the periodontitis group in comparison with the control group. In comparison with the control group, significantly lower levels of serum and salivary TAOC levels were observed in the periodontitis group. However, they did not observe any significant difference in the mean values of serum MDA levels. Overall, the oxidative stress was comparatively higher in the GP group in comparison with the $\mathrm{CP}$ group. On correlating the periodontal parameters and the oxidative parameters, significant positive and negative correlations were observed. From the results, the authors concluded that in the pathology of periodontitis, a significant role is played by elevated TOS and decreased TAOC. ${ }^{18}$

On comparing the mean salivary MDA, TOS, TAOC, and OSI levels in between the three study groups, significant results were obtained (Tables 2 and 3). Similar results were reported in the past literature. ${ }^{18}$ Baltacioğlu et $\mathrm{al}^{19}$ investigated the correlation between the TOS and receptor activator of nuclear factor- $\mathrm{\kappa B}$ ligand (RANKL) and osteoprotegerin (OPG) levels in CP and GP patients. They assessed 30 GP patients, $30 \mathrm{CP}$ patients, and 28 healthy controls. Automatic colorimetric method and
ELISA methods were used for the estimation of serum and GCF TOS, RANKL, and OPG levels. They observed that in comparison with the control group, the patients with periodontitis had higher value of mean serum and GCF levels of the above-mentioned parameters. From the results, they concluded that severity of periodontitis is closely related to the oxidative stress. Superoxide dismutase concentration, TOS, and MDA levels in periodontal patients were examined by Wei et al. ${ }^{20}$ They also examined the impact of periodontal therapy on the index levels in $\mathrm{CP}$ patients. After analyzing the $\mathrm{CP}$ patients and controls, they observed that in the periodontal region, LPO levels were significantly higher.

In another study conducted by $\mathrm{D}^{\prime}$ Aiuto et $\mathrm{al}^{21}{ }^{21} 145$ periodontitis patients and 56 healthy controls were evaluated. Assessment of diacron reactive oxygen metabolites (D-ROM), antioxidant potential, C-reactive protein, interleukin-6, and lipid profiles was done in all the patients at various time intervals. Higher D-ROM levels were observed in subjects with severe periodontitis. Their results depicted a positive correlation between periodontitis and oxidative stress.

\section{CONCLUSION}

Significantly higher amount of oxidative stress is found in periodontitis patients in comparison with healthy subjects. The GP patients are subjected to higher oxidative stress than $\mathrm{CP}$ patients. Therefore, these oxidative parameters do play a significant role in the pathologic profile of periodontitis. However, future studies are required with higher sample size and more number of parameters for better exploration of this field of periodontal medicine.

\section{REFERENCES}

1. Dede FÖ, Ozden FO, Avc1 B. 8-hydroxy-deoxyguanosine levels in gingival crevicular fluid and saliva in patients with chronic periodontitis after initial periodontal treatment. J Periodontol 2013 Jun;84(6):821-828.

2. Spickett CM. The lipid peroxidation product 4-hydroxy2-nonenal: advances in chemistry and analysis. Redox Biol 2013 Jan;1(1):145-152.

3. Chapple IL, Matthews JB. The role of reactive oxygen and antioxidant species in periodontal tissue destruction. Periodontol 20002007 Feb;43(1):160-232.

4. Hernandez M, Valenzuela MA, Lopez-Otin C, Alvarez J, Lopez JM, Vernal R, Gamonal J. Matrix metalloproteinase-13 is highly expressed in destructive periodontal disease activity. J Periodontol 2006 Nov;77(11):1863-1870.

5. Kinney JS, Ramseier CA, Giannobile WV. Oral fluid-based biomarkers of alveolar bone loss in periodontitis. Ann N Y Acad Sci 2007 Mar;1098:230-251.

6. Herr AE, Hatch AV, Throckmorton DJ, Tran HM, Brennan JS, Giannobile WV,Singh AK. Microfluidic immunoassays as rapid saliva-based clinical diagnostics. Proc Natl Acad Sci U S A 2007 Mar;104(13):5268-5273. 
7. Chrousos GP, Gold PW. The concepts of stress and stress system disorders. Overview of physical and behavioral homeostasis. JAMA 1992 Mar;267(9):1244-1252.

8. Sayre LM, Lin D, Yuan Q, Zhu X, Tang X. Protein adducts generated from products of lipid oxidation: focus on HNE and one. Drug Metab Rev 2006 Feb;38(4):651-675.

9. Armitage GC. Development of a classification system for periodontal diseases and conditions. Ann Periodontol 1999 Dec;4(1):1-6.

10. Page RC, Eke PI. Case definitions for use in populationbased surveillance of periodontitis. J Periodontol 2007 Jul;78 (7 Suppl):1387-1399.

11. Loe H, Silness J. Periodontal disease in pregnancy. I. Prevalence and severity. Acta Odontol Scand 1963 Dec;21:533-551.

12. Mühlemann HR, Son S. Gingival sulcus bleeding-a leading symptom in initial gingivitis. Helv Odontol Acta 1971 Oct;15(2):107-113.

13. Silness J, Loe H. Periodontal disease in pregnancy. II. correlation between oral hygiene and periodontal condition. Acta Odontol Scand 1964 Feb;22:121-135.

14. Akalin FA, Baltacioğlu E, Alver A, Karabulut E. Lipid peroxidation levels and total oxidant status in serum, saliva and gingival crevicular fluid in patients with chronic periodontitis. J Clin Periodontol 2007 Jul;34(7):558-565.

15. Sculley DV, Langley-Evans SC. Periodontal disease is associated with lower antioxidant capacity in whole saliva and evidence of increased protein oxidation. Clin Sci (Lond) 2003 Aug;105(2):167-172.

16. Young IS, Trimble ER. Measurement of malondialdehyde in plasma by high performance liquid chromatography with fluorimetric detection. Ann Clin Biochem 1991 Sep;28(Pt 5): 504-508.

17. Erel O. A new automated colorimetric method for measuring total oxidant status. Clin Biochem 2005 Dec;38(12):1103-1111.

18. Baltacıŏglu E, Yuva P, Aydın G, Alver A, Kahraman C, Karabulut E, Akalin FA. Lipid peroxidation levels and total oxidant/antioxidant status in serum and saliva from patients with chronic and aggressive periodontitis. Oxidative stress index: a new biomarker for periodontal disease? J Periodontol 2014 Oct;85(10):1432-1441.

19. Baltacioğlu E, Kehribar MA, Yuva P, Alver A, Atagün OS, Karabulut E, Akalin FA. Total oxidant status and bone resorption biomarkers in serum and gingival crevicular fluid of patients with periodontitis. J Periodontol 2014 Feb;85(2):317-326.

20. Wei D, Zhang XL, Wang YZ, Yang CX, Chen G. Lipid peroxidation levels, total oxidant status and superoxide dismutase in serum, saliva and gingival crevicular fluid in chronic periodontitis patients before and after periodontal therapy. Aust Dent J 2010 Mar;55(1):70-78.

21. D'Aiuto F, Nibali L, Parkar M, Patel K, Suvan J, Donos N. Oxidative stress, systemic inflammation, and severe periodontitis. J Dent Res 2010 Nov;89(11):1241-1246. 